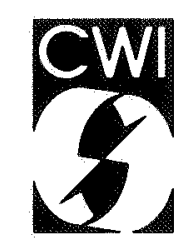

Centrum voor Wiskunde en Informatica Centre for Mathematics and Computer Science

M. Bezem

Semantics and consistency of rule-based expert systems 
The Centre for Mathematics and Computer Science is a research institute of the Stichting Mathematisch Centrum, which was founded on February 11, 1946, as a nonprofit institution aiming at the promotion of mathematics, computer science, and their applications. It is sponsored by the Dutch Government through the Netherlands Organization for the Advancement of Pure Research (Z.W.O.).

Copyright $\odot$ Stichting Mathematisch Centrum, Arnsterdam

6 gk $13,69 k 14,69 ; 4$ 


\title{
Semantics and Consistency of Rule-based Expert Systems
}

\author{
Marc Bezem \\ Centre for Mathematics and Computer Science \\ P.O. Box 4079, 1009 AB Amsterdam, The Netherlands
}

\begin{abstract}
Consistency of a knowledge-based system has become a topic of growing concern. Every notion of consistency presupposes a notion of semantics. We present a theoretical framework in which both the semantics and the consistency of a knowledge base can be studied. This framework is based on first order flat many-sorted predicate logic and is sufficiently rich to capture an interesting class of rule-based expert systems and deductive databases. We analyse the feasibility of the consistency test and prove that this test is feasible for knowledge bases in Horn format without quantification. This report is a revised and extended version of report CS-R8736. The extension concerns the use of expressions in rules.
\end{abstract}

1980 Mathematics Subject Classification: 68T30, 68T15.

1987 CR Categories: 1.2.3, 1.2.4, F.4.1.

Key Words \& Phrases: knowledge-based systems, rule-based expert systems, knowledge representation, consistency.

Note: The material for this article has been taken from the PRISMA documents P176 and P297. The research is part of the PRISMA project (PaRallel Inference and Storage MAchine), a joint effort with Philips Research Eindhoven, partially supported by the Dutch "Stimulerings-projectteam Informatica-onderzoek" (SPIN).

\section{INTRODUCTION}

The plan of this paper (a revised and extended version of $[B]$ ) is as follows. First the reader is introduced to the knowledge representation used in rule-based expert systems. We shall indicate some semantical problems in relation to this knowledge representation. Then we explain in an informal way how many-sorted predicate logic comes in. In the next section we describe syntax and semantics of many-sorted predicate logic. We assume some knowledge of first order logic. Thereafter we shall be able to characterize rule-based expert systems as first order theories. The Tarski semantics solves the semantical problems mentioned above. Furthermore we shall derive several results on decidability and consistency of rule-based expert systems. Unfortunately, some natural equality and ordering axioms are not in Horn format (see [Re] for a discussion on the domain closure axiom). Hence testing consistency with a standard theorem prover would be very inefficient. In the fourth section we describe a technical device, a certain kind of null value, which allows feasible consistency testing in the presence of equality and ordering axioms, which are not in Horn format. The underlying idea is partiality of functions, thus avoiding the search for consistent function values in a (possibly gigantic) product space. This kind of null value, being quite different from null values as described in [IL], appears to be new. In the last section we show how to extend our results to knowledge bases with arithmetical (and other) expressions in the conditions of the rules. We shall focus our attention on rule-based expert systems, but the techniques can also be applied to deductive databases with functional dependencies.

Report CS-R8824

Centre for Mathematics and Computer Science

P.O. Box 4079, 1009 AB Amsterdam, The Netherlands 


\title{
1. RULE-BASED EXPERT SYSTEMS
}

1.1. In rule-based expert systems shells such as EMYCIN [BS] or DELFI2 [L], knowledge about some specific domain can be expressed in facts and in rules of the form

\section{IF antecedent THEN consequent.}

Facts are so-called object-attribute-value triples, or $\langle o, a, v\rangle$ triples for short. The antecedent of a rule is a conjunction of disjunctions of conditions, and conditions are definite statements, such as same, notsame and less than, about $\langle o, a, v\rangle$ triples. We restrict ourselves to rules having as consequent a conjunction of conclusions of the form conclude $\langle o, a, v\rangle$. In most cases so-called certainty factors are associated with the facts and the rules. Certainty factors range from 1.00 (definitely true) to -1.00 (definitely false). The certainty factor of a fact expresses a measure of certainty about that fact, whereas the certainty factor of a rule scales the measure of certainty about the consequent with respect to the measure of certainty about the antecedent. In DELFI2 an object tree (called context tree in MYCIN) is used to state properties of and relations between different objects, which cannot be expressed by the rules. The nodes of this tree are objects, labeled by their attributes and respective domains of values. The path from a node to the root of this tree constitutes the context of that node. In other words: the objects occurring in the subtree of a node are sub-objects of the object belonging to that node. Furthermore it is stated in the object tree whether an attribute is singlevalued or multivalued.

The interpretation of the knowledge in rule-based expert systems is more operational than declarative: same $\langle o, a, v\rangle$ is true if and only if $\langle o, a, v\rangle$ occurs as fact (with certainty factor $\rangle 0.2$ ), conclude $\langle o, a, v\rangle$ has the effect that $\langle o, a, v\rangle$ is added as fact (with appropriate certainty factor), and if the antecedent of a rule evaluates to true, then that rule may be fired, i.e. all conclusions occurring in the consequent are executed. We remark that same $\langle o, a, v\rangle$ and conclude $\langle o, a, v\rangle$ have the same declarative meaning as the fact $\langle o, a, v\rangle$, i.e. attribute $a$ of object $o$ has value $v$.

1.2. Consider the following real-life example extracted from HEPAR, an expert system for the diagnosis of liver and biliary disease, built with DELFI2.

$$
\begin{aligned}
& \text { IF same 〈patient,complaint,colicky pain〉 } \\
& \text { THEN conclude 〈patient,pain,colicky〉 }
\end{aligned}
$$

IF same (patient,abd pain,yes〉 AND

same 〈pain,character,continuous〉

THEN conclude 〈patient,pain, colicky〉 $(-1.00)$

\author{
IF same (patient,complaint,abdominal pain) OR \\ same 〈patient,pain, colicky) \\ THEN conclude 〈patient,abd pain,yes〉 (1.00)
}

These three rules (from a rule base consisting of over 400 rules) show two objects, patient and pain, four attributes, namely complaint, pain and abd pain of patient and character of pain, as well as several values.

1.3. A first observation which can be made is the following. Five items in the rules above refer to pain: the values colicky_pain and abdominal pain, the attributes pain and abd pain of the object patient, and the object pain, which is a sub-object of patient, as stated by the object tree of HEPAR. 
The interrelations between these items do not seem to be expressible by the formalism.

A second observation on the three rules above is their inconsistency in the presence of the facts 〈patient,complaint, colicky pain〉 (1.00) and 〈pain,character,continuous〉 (1.00). The inference engine reasons backwards, using a simple loop check to prevent infinite looping. Depending on the presence of the fact 〈patient, complaint,abdominal pain〉 (1.00), the inference engine did or did not hit upon the contradiction 〈patient,pain, colicky〉 $(1.00$ and -1.00$)$. In both cases the contradiction was completely ignored.

These observations show a defect of the knowledge representation used in rule-based expert systems, namely the lack of a clear semantics.

1.4. Basically our approach amounts to interpreting $\langle o, a, v\rangle$ by $a(o, v)$ in the multivalued, and by $a(o)=v$ in the singlevalued case. Here $o$ and $v$ are constants for elements of a domain $O$ of objects and a domain $V$ of values. In the multivalued case $a$ denotes a relation, i.e. a subset of $O \times V$, and in the singlevalued case a function from $O$ to $V$. If $o$ is a sub-object of $o^{\prime}$, then $o^{\prime}$ is added as argument of a.

1.5. The following examples show how to extend our interpretation of $\langle o, a, v\rangle$ triples to atoms.

$$
\text { conclude 〈patient,complaint,abdominal pain〉 }
$$

becomes

$$
\text { complaint (patient,abdominal pain) }
$$

less_than 〈patient, temperature,36.8)

becomes

$$
\text { temperature (patient) }<36.8
$$

same 〈pain,character, continuous〉

becomes

$$
\text { character }(\text { patient, pain })=\text { continuous }
$$

Note that the fact that pain is a sub-object of patient is expressed by adding patient as an argument of the function character. Another example, based on the rules of 1.2, is to be found in 4.6.

We feel that the existing formalisms for handling uncertainty are unsatisfactory. As we do not have a good alternative we leave the subject aside.

1.6. Under the interpretation described above, a rule-based expert system becomes a theory in first order many-sorted predicate logic, in short: a many-sorted theory. To keep this paper self-contained we give a short, introductory description of the syntax and semantics of many-sorted predicate logic in the next section. In Section 3 we shall characterize expert systems as many-sorted theories of a certain type. This approach has the following advantages:

- The declarative semantics of the expert system becomes perfectly clear, being the Tarski semantics of the associated many-sorted theory.

- Logical concepts such as consequence, consistency and decidability get a clear meaning in relation to the expert system.

- Theorem proving techniques for testing consistency, such as resolution, become available for the expert system. 
The choice for many-sorted instead of one-sorted logic is motivated as follows:

- It is natural to make a distinction between numerical data (ordered by $<$ ) and symbolic data (usually unordered).

- Subdividing a set of constants into sorts and typing the predicate and function symbols considerably reduces the number of well-formed formulas, which is important for debugging large knowledge bases.

- In the presence of variables the many-sorted approach imposes restrictions on unification, which considerably reduces the resolution search space (see for example [W]). We shall not make use of this advantage in the present paper.

\section{ManY-Sorted Predicate Logic}

2.1. The syntax of many-sorted predicate logic extends the syntax of ordinary, one-sorted, predicate logic by having a set of sorts $\Sigma$, instead of just one sort. Moreover we have variables $x_{i}^{\sigma}$ and constants $c_{i}^{\sigma}$ for all sorts $\sigma \in \Sigma$. Furthermore we have function symbols $f_{i}^{\sigma_{1} \times \cdots \times \sigma_{m} \rightarrow \sigma_{0}}(m>0)$, where the notion of type $\sigma_{1} \times \cdots \times \sigma_{m} \rightarrow \sigma_{0}$ replaces the notion of arity from the one-sorted case. We also have proposition symbols $p_{i}$ and predicate symbols $P_{i}^{\sigma_{1} \times \cdots \times \sigma_{m}}(m>0)$ of type $\sigma_{1} \times \cdots \times \sigma_{m}$. Terms are formed from variables and constants by function application (respecting the sorts). Atoms are either proposition symbols or the application of a predicate symbol to terms of appropriate sorts. With the help of propositional connectives and quantifiers, atoms are combined into formulas. The sets $\Sigma$, CONS, FUNC, PROP and PRED of, respectively, sorts, constants, function symbols, proposition symbols and predicate symbols, form together the similarity type of a specific many-sorted predicate calculus. For practical reasons we assume that the similarity type is finite.

2.2. A many-sorted structure $\Re$ consists of:

(a) A non-empty set $A_{\sigma}$ for each $\sigma \in \Sigma$, called the domain of sort $\sigma$.

(b) For each constant $c_{i}^{\sigma}$ an element $\bar{c}_{i} \in A_{\sigma}$.

(c) For each function symbol $f_{i}^{\sigma_{1} \times \cdots \times \sigma_{m} \rightarrow \sigma_{0}}$ a mapping $\bar{f}_{i}: A_{\sigma_{1}} \times \cdots \times A_{\sigma_{m}} \rightarrow A_{\sigma_{0}}$.

(d) For each proposition symbol $p_{i}$ a truth value $\bar{p}_{i}$.

(e) For each predicate symbol $P_{i}^{\sigma_{1}} \times \cdots \times \sigma_{\sigma_{m}}$ a mapping $\bar{P}_{i}: A_{\sigma_{1}} \times \cdots \times A_{\sigma_{m}} \rightarrow\{T R U E, F A L S E\}$.

2.3. An assignment in $\mathfrak{R}$ is a mapping $a$ assigning to each variable $x_{i}^{\sigma}$ an element $a\left(x_{i}^{\sigma}\right)$ of $A_{\sigma}$.

2.4. The interpretation in $\Re$ of a term $t$ under an assignment $a$, denoted by $I_{a}^{\Re(}(t)$ or $\bar{t}$ for short, is inductively defined as follows:

(a) $\overline{x_{i}^{\sigma}}=a\left(x_{i}^{\sigma}\right)$

(b) $\bar{c}_{i}^{\mathbf{\sigma}}=\bar{c}_{i}$

(c) $\frac{f_{i}^{\sigma_{1} \times \cdots \times \sigma_{m} \rightarrow \sigma_{0}}\left(t_{1}, \ldots, t_{m}\right)}{=}=\bar{f}_{i}\left(\bar{t}_{1}, \ldots, \bar{t}_{m}\right)$

The truth value in $\Re$ of an atom $P_{i}^{\sigma_{1} \times \cdots \times \sigma_{m}}\left(t_{1}, \ldots, t_{m}\right)$ under an assignment $a$ is given by $\bar{P}_{i}\left(\bar{t}_{1}, \ldots, \bar{t}_{m}\right)$.

2.5. The truth value in $\Re$ of a formula $F$ under an assignment $a$, denoted by $\Upsilon_{a}^{P \pi}(F)$, is inductively defined as follows:

(a) If $F$ is an atom, then $\mathcal{T}_{a}^{\text {श }}(F)$ is given by 2.4 .

(b) $\Upsilon_{a}^{\text {PR }}$ respects the truth tables of the propositional connectives.

(c) $\Upsilon_{a}^{\rho R}\left(\forall x_{i}^{\sigma} F\right)=T R U E$ if and only if for all assignments $a^{\prime}$, which differ at most on $x_{i}^{\sigma}$ from $a$, we have $\mathcal{T}_{a^{\prime}}(F)=$ TRUE.

(d) $\Upsilon_{a}^{\Re R}\left(\exists x_{i}^{\sigma} F\right)=$ TRUE if and only if there exists an assignment $a^{\prime}$, which differs at most on $x_{i}^{\sigma}$ from $a$, such that ${ }^{\prime} a^{\prime}(F)=T R U E$. 
2.6. A formula $F$ is true in $\Re$, denoted by $k_{\Re} F$, if $\gamma_{a}^{\Re}(F)=T R U E$ for all assignments $a$.

2.7. A sentence (or closed formula) is a formula without free variables (i.e. variables which are not bound by a quantifier). It will be clear that for sentences $S$ the truth value $\tau_{a}^{g / R}(S)$ does not depend on the assignment $a$. As a consequence we have either $k_{\Re} S$ or $k_{\Re} S$, for every sentence $S$. Let SENT denote the set of sentences.

2.8. Let $\Gamma \subset$ SENT. $\Re$ is called a model for $\Gamma$, denoted by $k_{\pi} \Gamma$, if $k_{\Re} S$ for all $S \in \Gamma$.

2.9. $S \in$ SENT is called a (semantical) consequence of (or implied by) $\Gamma \subset$ SENT if for all manysorted structures $\Re$ we have: if $k_{\Re R} \Gamma$, then $k_{\Re} S$. This will be denoted by $\Gamma \vDash S$ (or $\vDash S$ if $\Gamma$ is empty). Furthermore we define the theory of $\Gamma$ as the set $T h(\Gamma)=\{S \in \operatorname{SENT} \mid \Gamma \vDash S\}$.

2.10. $\Gamma \subset$ SENT is called consistent if $\Gamma$ has a model. $T h(\Gamma)$ is called decidable if there exists a mechanical decision procedure to decide whether a given sentence $S$ is a semantical consequence of $\Gamma$ or not.

2.11. Two many-sorted structures are called elementarily equivalent if exactly the same sentences are true in both structures.

\subsection{REMARKS.}

2.12.1. We refrain from giving an axiomatization of many-sorted predicate logic since our main concern will be model theory. Most textbooks on mathematical logic provide a complete axiomatization of ordinary (one-sorted) predicate logic. It suffices to generalize the quantifier rules in order to obtain an axiomatization of flat many-sorted predicate logic.

2.12.2. Of course, one-sorted predicate logic is a special case of many-sorted predicate logic. As a consequence, the latter is as undecidable as the former. More precisely: $₹ S$ is undecidable, provided that the similarity type is rich enough ( CHURCH, TURING, 1936, see also [M, 16.58]).

2.12.3. Conversely, many-sorted predicate logic can be embedded in one-sorted predicate logic by adding unary predicate symbols $S^{\sigma}(x)$, expressing that $x$ is of sort $\sigma$, and replacing inductively in formulas $\forall x_{i}^{\sigma} F\left(\right.$ resp. $\left.\exists x_{i}^{\sigma} F\right)$ by $\forall x\left(S^{\sigma}(x) \rightarrow F\right)$ (resp. $\exists x\left(S^{\sigma}(x) \wedge F\right)$ ). Let $A^{\prime}$ be the one-sorted sentence obtained from $A \in \mathrm{SENT}$ in this way. It can be proved (see [M]) that $k A$ if and only if $\Gamma \vDash A^{\prime}$, where $\Gamma=\left\{\exists x S^{\sigma}(x) \mid \sigma \in \Sigma\right\}$ expresses the fact that the domains are non-empty. This embedding allows us to generalize immediately many results on one-sorted predicate calculus to the many-sorted case (e.g. the compactness theorem). We shall not make use of this possibility in the present paper.

\section{Rule-Based Expert Systems as Many-Sorted Theories}

3.0. We propose the following terminology for certain kinds of many-sorted theories:

- Indexed propositional expert systems.

- Universally quantified expert systems.

3.1. An indexed propositional expert system is a many-sorted theory axiomatized by:

(a) Explicit axioms (the rule base and the fact base), which are boolean combinations of atoms of the form $P^{\sigma_{1} \times \cdots \times \sigma_{m}}\left(c, \ldots, c^{\prime}\right)$ or of the form $f^{\left(\sigma_{1} \times \cdots \times \sigma_{m}\right) \rightarrow \sigma_{0}}\left(c, \ldots, c^{\prime}\right)={ }_{\sigma_{0}} c^{\prime \prime}\left(\right.$ resp. $\left.<_{\sigma_{0}} c^{\prime \prime},>_{\sigma_{0}} c^{\prime \prime}\right)$, with constants $c, \ldots, c^{\prime}, c^{\prime \prime}$ of appropriate sorts. Such atoms (here and below called constant-atoms, or $c$-atoms for short) may be viewed as indexed propositions, which explains the name. Note that we conform to the convention to write $=,<$ and $>$ as infix predicates. 
(b) Implicit axioms for equality of each sort and ordering of each sort for which an ordering is appropriate. The axioms for $=_{\sigma}$, equality of sort $\sigma$, are (loosely omitting sort super- and subscripts):

$$
\begin{aligned}
& \forall x(x=x), \\
& \forall x_{1}, x_{2}\left(x_{1}=x_{2} \rightarrow x_{2}=x_{1}\right), \\
& \forall x_{1}, x_{2}, x_{3}\left(\left(x_{1}=x_{2} \wedge x_{2}=x_{3}\right) \rightarrow x_{1}=x_{3}\right), \\
& \forall x_{1}, x_{2}\left(\left(x_{1}=x_{2} \wedge F\left(x_{1}\right)\right) \rightarrow F\left(x_{2}\right)\right) \text { for formulas } \mathrm{F}, \\
& \neg c_{i}=c_{j} \text { for } 0 \leqslant i \neq j \leqslant n, \\
& \forall x\left(x=c_{0} \vee \cdots \vee x=c_{n}\right), \text { the domain closure axiom. }
\end{aligned}
$$

These axioms express that $=$ is a congruence relation on a finite domain, where every element has exactly one name. Let $E Q$ denote the set of equality axioms for all sorts $\sigma$, then we have by definition that either $E Q \vDash c_{i}=c_{j}$ or $E Q \vDash \neg c_{i}=c_{j}$ for all $\mathrm{i}, \mathrm{j}$. The axioms for $<$ and $>$ are:

$$
\begin{aligned}
& \forall x_{1}, x_{2}, x_{3}\left(\left(x_{1}<x_{2} \wedge x_{2}<x_{3}\right) \rightarrow x_{1}<x_{3}\right), \\
& \forall x(\neg x<x), \\
& \forall x_{1}, x_{2}\left(x_{1}<x_{2} \vee x_{1}=x_{2} \vee x_{2}<x_{1}\right) \text { for sorts which are totally ordered, } \\
& \forall x_{1}, x_{2}\left(x_{1}<x_{2} \leftrightarrow x_{2}>x_{1}\right), \\
& \text { a subset } \Delta \text { of }\left\{c_{i}<c_{j} \mid 0 \leqslant i \neq j \leqslant n\right\} \cup\left\{\neg c_{i}<c_{j} \mid 0 \leqslant i, j \leqslant n\right\} \text { (see below). }
\end{aligned}
$$

These axioms express that $<$ is a transitive, irreflexive and (possibly) total ordering with inverse $>$. Let $O$ denote the set of ordering axioms. We require that $\Delta$ is such that either $O \vDash c_{i}<c_{j}$ or $O \vDash \neg c_{i}<c_{j}$, for all i,j. It follows in particular that $E Q \cup O$ is consistent.

The idea behind the implicit axioms is that $=$ and $<$ are provided by the system and have a fixed meaning, whereas the other predicates are user-defined. We put $I A=E Q \cup O$, so $I A$ denotes the set of implicit axioms.

3.2. A universally quantified expert system differs from an indexed propositional one by allowing not only constants, but also variables in the explicit axioms. All explicit axioms are assumed to be universally closed.

3.3. Among the theories that do not fall under 3.1 and 3.2 are many theorem provers. A theorem prover might be an undecidable theory. The theoretical observations below show that, from a logical point of view, expert systems as defined in 3.1 and 3.2 are very simple, decidable theories.

3.4. LEMMA. For every $S \in \mathrm{SENT}$ there exists a boolean combination $S^{\prime}$ of closed atoms such that $E Q \vDash S \leftrightarrow S^{\prime}$

Proof. Replace inductively every subformula $\forall x F(x)$ by $F\left(c_{0}\right) \wedge \cdots \wedge F\left(c_{n}\right)$ and $\exists x F(x)$ by $F\left(c_{0}\right) \vee \cdots \vee F\left(c_{n}\right)$ where $\forall x\left(x=c_{0} \vee \cdots \vee x=c_{n}\right) \in E Q$.

3.5. LEMMA. For every closed atom $A$ there exists a boolean combination $A^{\prime}$ of c-atoms such that $E Q \vDash A \leftrightarrow A^{\prime}$.

Proof by giving a typical example. Let $A$ be for instance $P\left(f\left(f^{\prime}\left(c^{\sigma_{0}}\right), c^{\sigma_{1}}\right), c^{\sigma_{2}}\right)$ with $f$ of type $\sigma_{2} \times \sigma_{1} \rightarrow \sigma_{0}$ and $f^{\prime}$ of type $\sigma_{0} \rightarrow \sigma_{2}$. Let $A^{\exists}$ be the sentence $\exists x^{\sigma_{2}} \exists y^{\sigma_{0}}\left[f^{\prime}\left(c^{\sigma_{0}}\right)=x^{\sigma_{2}} \wedge f\left(x^{\sigma_{2}}, c^{\sigma_{1}}\right)=y^{\sigma_{0}} \wedge P\left(y^{\sigma_{0}}, c^{\sigma_{2}}\right)\right]$. Now apply Lemma 3.4 to $A^{\exists}$ and obtain $A^{\prime}$. 
3.6. Both lemmas above can cause combinatorial explosions. Therefore they are only of theoretical use. They tell us that, in the presence of $E Q$, boolean combinations of $c$-atoms have the same expressive power as full many-sorted predicate logic.

3.7. LEMMA. If $E Q \subset \Gamma \subset \mathrm{SENT}$, then every model of $\Gamma$ is elementarily equivalent to a model whose domains consist of exactly the interpretations of all constants.

Proof. Let $\Re$ be a model of $\Gamma$ with $E Q \subset \Gamma \subset$ SENT. By $E Q$ the interpretations of the equality predicates in OR are equivalence relations which are congruences with respect to the interpretation of all other predicate and function symbols in $\Re$. It follows that $\mathscr{N}$ and $\mathscr{N} /=$, the quotient structure of $\Re$ modulo equality, are elementarily equivalent. By the axioms $\forall x\left(x=c_{0} \vee \cdots \vee x=c_{n}\right)$ and $\neg c_{i}=c_{j}(i \neq j)$ from $E Q$, it follows that the domains of $\mathscr{T} /=$ consist of exactly the interpretations of all constants.

\subsection{LEMma. If $E Q \subset \Gamma \subset \mathrm{SENT}$, then $T h(\Gamma)$ is decidable.}

Proof. Let $\Gamma$ be such that $E Q \subset \Gamma \subset$ SENT. Then we can apply Lemma 3.7 and observe that there are just finitely many non-isomorphic $\Re U=$ 's. In other words: up to dividing out $=$ and identifying individuals with the same name there are just finitely many different models of $\Gamma$. Moreover all models of $\Gamma$ are finite. Hence we can, for any given $S \in$ SENT, test in finite time whether $S$ holds in all models of $\Gamma$ or not.

\section{TESTING CONSISTENCY}

4.1. Definition 2.10 and Lemma 3.7 suggest the following procedure for testing the consistency of theories $\Gamma$ with $E Q \subset \Gamma$ : generate all many-sorted structures whose domains consist of exactly the interpretations of all constants, and test each time whether the many-sorted structure is a model of $\Gamma$ or not. This procedure is in general not feasible. A first step towards a feasible consistency test is the alternative characterization of consistency for indexed propositional expert systems, described in the following paragraphs.

Let $\Gamma$ be an axiomatization of an indexed propositional expert system (see 3.1). Let $P_{1}, \ldots, P_{m}$ be a list of all $c$-atoms of the form $P\left(c, \ldots, c^{\prime}\right)$ occurring in $\Gamma$, and let $t_{1}, \ldots, t_{n}$ list all terms $f\left(c, \ldots, c^{\prime}\right)$ occurring in $\Gamma$. The idea behind the following construction is that it is not necessary to have an entire many-sorted structure to be able to interpret $\Gamma$.

Let $A_{\sigma}=\left\{c_{0}^{\sigma}, \ldots, c_{n}^{\sigma}\right\}$ be the set of all constants of sort $\sigma \in \Sigma$. Equality of sort $\sigma$ is interpreted by syntactical identity on $A_{\sigma}$. Then all equality axioms from $E Q$ are satisfied. The ordering (if any) on $A_{\sigma}$ is induced by $O$, i.e. $c_{i}<c_{j}$ if and only if $O \vDash c_{i}<c_{j}$. We need the following notions:

- A truth valuation of $P_{1}, \ldots, P_{m}$ is an assignment of either TRUE or FALSE to each $P_{i}(1 \leqslant i \leqslant m)$.

- A valuation of $t_{1}, \ldots, t_{n}$ is an assignment of a unique element of the appropriate domain $A_{\sigma}$ to each $t_{j}$ $(1 \leqslant j \leqslant n)$. It will be clear that a truth valuation of $P_{1}, \ldots, P_{m}$ and a valuation of $t_{1}, \ldots, t_{n}$ suffice for an interpretation of $\Gamma$. Each model of $\Gamma$ yields a truth valuation of $P_{1}, \ldots, P_{m}$ and a valuation of $t_{1}, \ldots, t_{n}$. Conversely, each truth valuation of $P_{1}, \ldots, P_{m}$ and valuation of $t_{1}, \ldots, t_{n}$ for which every explicit axiom of $\Gamma$ is true, can be extended in an arbitrary way to a many-sorted structure $\Re \vDash \Gamma$. Thus we have the following

THEOREM. Let conditions be as above. Then we have: $\Gamma$ is consistent if and only if there exists a truth valuation of $P_{1}, \ldots, P_{m}$ and a valuation of $t_{1}, \ldots, t_{n}$ for which every explicit axiom of $\Gamma$ is true.

4.2. Theorem 4.1 suggests a simple algorithm for testing the consistency of an indexed propositional expert system $\Gamma$ : generate all valuations and truth valuations and test each time whether the explicit 
axioms of $\Gamma$ are satisfied or not. This clearly leads to combinatorial explosions. A second step towards a feasible consistency test is imposing language restrictions on our expert systems.

A common language restriction is Horn format. A clause is a finite disjunction of atoms and negations of atoms (so called positive and negative literals). A conjunctive normal form is a finite conjunction of clauses. A Horn clause is a clause containing at most one positive literal. A unit clause is a clause consisting of one positive literal. The connection between Horn clauses and production rules is easily seen by the equivalence of $\left(A_{1} \wedge \cdots \wedge A_{k}\right) \rightarrow B$ and $A_{1}^{-} \vee \cdots \vee A_{k}^{-} \vee B^{+}$, where the superscripts + and - denote whether a literal occurs positively or negatively. However, the implicit axioms $\forall x\left(x=c_{0} \vee \cdots \vee x=c_{n}\right)$ and $\forall x_{1}, x_{2}\left(x_{1}<x_{2} \vee x_{1}=x_{2} \vee x_{2}<x_{1}\right)$ are not Horn clauses. As a consequence we can only require the explicit axioms to be Horn clauses. This is not sufficient for feasible consistency checking, as will be demonstrated in the next paragraph. The idea is to reduce the satisfiability problem for propositional logic, which is known to be NP-complete (see [GJ]), to the consistency problem of indexed propositional expert systems, all whose explicit axioms are Horn clauses.

Let $\sigma$ be a sort with exactly two constants: $c_{0}^{\sigma}$ and $c_{1}^{\sigma}$. Then we have $\forall x\left(x=c_{0} \vee x=c_{1}\right) \in E Q$. For any propositional atom $A$, let $f_{A}$ be a function symbol of type $\sigma \rightarrow \sigma$. It is not difficult to see that

$$
\left\{\neg f_{A}\left(c_{0}\right)=c_{0} \vee A, f_{A}\left(c_{0}\right)=c_{0} \vee \neg A\right\} \cup E Q \vDash\left(\neg f_{A}\left(c_{0}\right)=c_{1}\right) \leftrightarrow A .
$$

So the positive literal $A$ is equivalent to the negative literal $\neg f_{A}\left(c_{0}\right)=c_{1}$ in any $\Gamma$ containing the two Horn clauses $\neg f_{A}\left(c_{0}\right)=c_{0} \vee A$ and $f_{A}\left(c_{0}\right)=c_{0} \vee \neg A$ and the equality axioms $E Q$. Let $C$ be any propositional conjunctive normal form. Let $\Gamma_{C}$ be the indexed propositional expert system with explicit axioms $\neg f_{A}\left(c_{0}\right)=c_{0} \vee A$ and $f_{A}\left(c_{0}\right)=c_{0} \vee \neg A$ for all positive literals $A$ occurring in $C$. Then $\Gamma_{C}$ is consistent and satisfies $\Gamma_{C} \vDash C \leftrightarrow H_{C}$, where $H_{C}$ is the set (conjunction) of Horn clauses obtained from $C$ by replacing all positive literals $A$ by their equivalent negative literal. Moreover we have that $C$ is satisfiable if and only if $\Gamma_{C} \cup H_{C}$ is consistent. This yields a polynomial reduction of the satisfiability problem for propositional logic to the consistency problem of indexed propositional expert systems, all whose explicit axioms are Horn clauses. A similar reduction could be established using $\forall x_{1}, x_{2}\left(x_{1}<x_{2} \vee x_{1}=x_{2} \vee x_{2}<x_{1}\right)$ instead of $\forall x\left(x=c_{0} \vee x=c_{1}\right)$.

4.3. In the previous subsection we showed that testing consistency is NP-hard without further language restrictions. The problem is to specify language restrictions, which are strong enough to guarantee a feasible consistency test, and still allow enough expressivity for some given application. This third and final step towards a feasible consistency test will be achieved in the following theorem by the introduction of constants undefined, which are different from (wrt. $=$ ) and incomparable with (wrt. <) any other constant. These constants are used for the simulation of partiality of functions, thus avoiding the search for consistent function values in a (possibly gigantic) product space of sorts. Further intuition will be given in 5.4.1.

THEOREM. Let $\Gamma$ be the axiomatization of an indexed propositional expert system satisfying the following conditions:

(I) All explicit axioms of $\Gamma$ are Horn clauses.

(2) For every sort $\sigma \in \Sigma$ there exists at least one constant, which does not occur in the explicit axioms of $\Gamma$. Such a constant, say $c_{0}^{\sigma}$, will be denoted by undefined ${ }^{\sigma}$. So we have:

$\forall x\left(x=\right.$ undefined $\left.\vee x=c_{1} \vee \cdots \vee x=c_{n}\right) \in E Q$ and $\neg$ undefined $=c_{i} \in E Q$ for all $1 \leqslant i \leqslant n$.

(3) For every sort $\sigma \in \Sigma$ the ordering (if any) of sort $\sigma$ is partial with respect to undefined ${ }^{\sigma}$. More precisely: we require that $O \vDash \neg$ undefined $\left\langle c_{i}\right.$ and $O \vDash \neg$ undefined $>c_{i}$ for all $1 \leqslant i \leqslant n$.

(4) The orderings $<$ and $>$ do not occur in the positive literals occurring in the explicit axioms of $\Gamma$.

Then the consistency of $\Gamma$ can be tested in polynomial time. 
Proof. List the explicit axioms of $\Gamma$ as follows (the super- and subscripted capitals denote $c$-atoms):

$$
\begin{aligned}
& A_{1}^{+} \\
& \cdot \\
& A_{p}^{+} \\
& C_{1,1}^{-} \vee \cdots \vee C_{1, n_{1}}^{-} \vee D_{1}^{+} \\
& \cdot \\
& \cdot \\
& C_{q, 1}^{-} \vee \cdots \vee C_{q, n_{q}}^{-} \vee D_{q}^{+} \\
& B_{1,1}^{-} \vee \cdots \vee B_{1, m_{1}}^{-} \\
& \cdot \\
& \cdot \\
& B_{r, 1}^{-} \vee \cdots \vee B_{r, m_{r}}^{-}
\end{aligned}
$$

Now apply the following algorithm, which is in fact a special case of hyper-resolution (see [R]). Under the conditions of the theorem, positive literals are either of the form $P\left(c, \ldots, c^{\prime}\right)$, or of the form $f\left(c, \ldots, c^{\prime}\right)=c^{\prime \prime}$. The current set of unit clauses is denoted by $U_{s}$. Anticipating the generalization in Section 5 the formulation of the algorithm is slightly more general than necessary: "EQ $\cup U_{s}$ is consistent" simply says that we do not have $f\left(c, \ldots, c^{\prime}\right)=c_{i} \in U_{s}$ and $f\left(c, \ldots, c^{\prime}\right)=c_{j} \in U_{s}$ with $i \neq j$, and " $c$-atom $X$ is implied by $I A \cup U_{s}$ " is the case if either $X \in U_{s}$, or $X$ is of the form $f\left(c, \ldots, c^{\prime}\right)<c_{i}$ (resp. $\left.>c_{i}\right)$ and we have $f\left(c, \ldots, c^{\prime}\right)=c_{j} \in U_{s}$ with $O \vDash c_{j}<c_{i}$ (resp. $O \vDash c_{j}>c_{i}$ ).

$\left({ }^{*}\right.$ generation of unit clauses *)

$$
\begin{aligned}
& U_{s}:=\left\{A_{1}^{+}, \cdots, A_{p}^{+}\right\} ; \\
& U_{\text {new }}:=\{\}
\end{aligned}
$$

\section{REPEAT}

$$
\begin{aligned}
& U_{s}:=U_{s} \cup U_{\text {new }} ; \\
& U_{\text {new }}:=\{\} \\
& I F E Q \cup U_{s} \text { is consistent }
\end{aligned}
$$

\section{THEN}

FOR each clause $C_{i, 1}^{-} \vee \cdots \vee C_{i, n_{i}}^{-} \vee D_{i}^{+}$

DO

cancel all $C_{i, j}^{-}$such that $C_{i, j}^{+}$is implied by $I A \cup U_{s}$;

IF all negative literals of the clause are cancelled

THEN $U_{\text {new }}:=U_{\text {new }} \cup\left\{D_{i}^{+}\right\}$

$O D$

UNTIL

$$
U_{\text {new }} \subset U_{s}
$$


( ${ }^{*}$ test if the unit clauses form a model *)

IF $E Q \cup U_{s}$ is consistent $A N D$ there exists no clause $B_{i, 1}^{-} \vee \cdots \vee B_{i, m_{i}}^{-}$such that all $B_{i, j}^{+}$are implied by $I A \cup U_{s}$

THEN $\Gamma$ is consistent

ELSE $\Gamma$ is inconsistent

The algorithm terminates since the number of literals is strictly decreasing. By the cancellations the explicit axioms of $\Gamma$ are transformed into an equivalent set of Horn clauses. The consistency in the THEN-part can be seen as follows. First note that $E Q \cup U_{s}$ is consistent. Secondly, every clause not in $U_{s}$ contains a negative literal $L^{-}$whose complement $L^{+}$is not implied by $I A \cup U_{s}$. It can happen that $I A \cup U_{s}$ implies $L^{-}$(for example $f(c)=2 \in U_{s}, L^{-}=\neg f(c)<1,1<2 \in \Delta \subset O$ ). Then every clause containing $L^{-}$holds in any model of $I A \cup U_{s}$. If neither $L^{-}$nor $L^{+}$is implied by $I A \cup U_{s}$, then we have either $L^{+}=P\left(c, \ldots, c^{\prime}\right) \notin U_{s}$, or $L^{+}$contains a function term $f\left(c, \ldots, c^{\prime}\right)$ such that $f\left(c, \ldots, c^{\prime}\right)=c^{\prime \prime} \in U_{s}$ for no constant $c^{\prime \prime}$. We define the following (truth-) valuation (see 4.1):

$$
\begin{aligned}
P_{i} & = \begin{cases}T R U E & \text { if } P_{i} \in U_{s} \\
F A L S E & \text { otherwise }\end{cases} \\
t_{j} & =\left\{\begin{array}{l}
c \text { if } t_{j}=c \in U_{s} \\
\text { undefined otherwise }
\end{array}\right.
\end{aligned}
$$

The valuation is well-defined since $E Q \cup U_{s}$ is consistent. By the properties of the constants undefined (conditions (2) and (3) of the theorem), the literals $L^{-}$mentioned above are TRUE in the (truth-) valuation. Hence every clause containing $L^{-}$is TRUE in the above (truth-) valuation, which constitutes (in the sense of Theorem 4.1) a model of the entire set of clauses. The algorithm is clearly quadratic in the number of occurrences of literals. To get the consistency of $\Gamma$ in complexity class $P$ we tacitly assumed that the primitive operations used in the algorithm above are in P.

\section{REMARKS.}

4.3.1. As follows by close inspection of the proof above, it would suffice to require the following weakening of condition (2): for every sort $\sigma$ for which a function symbol $f$ of type $\ldots \rightarrow \sigma$ occurs in a negative literal occurring in the explicit axioms of $\Gamma$, there exists at least one constant which does not occur on the right-hand side of an equation occurring in such a literal. However, we think it is more systematic to require condition (2) as it stands. In practice the constants undefined are simply added to every domain of constants occurring in the knowledge base.

4.3.2. Condition (4) can not be missed, which can be seen as follows. Assume for some sort $\sigma$ we have exactly three constants different from undefined, which are totally ordered by $c_{1}<c_{2}<c_{3}$. Then the unit clause $f(c)>c_{1}$ is equivalent to $f(c)=c_{2} \vee f(c)=c_{3}$, which enables a similar construction as in the third paragraph of 4.2 .

4.3.3. In view of condition (1), occurrences of notsame $\langle o, a, v\rangle$ in the antecedent of a rule can be problematic, since negative conditions in a rule result in positive literals in the clausal form. This problem can be overcome by postponing the consistency test until these occurrences evaluate to either TRUE or FALSE (e.g. after querying the user). Then the knowledge base can be transformed into an equivalent knowledge base satisfying (1). An alternative would be to allow clauses with more than one positive literal and to apply the following lemma. Of course the consistency test then becomes in general NP-hard, the worst-case time complexity doubles with every application of the lemma and we can only hope that practical cases are not the worst. 
LEMMA. Let $\Gamma$ be an axiomatization of an indexed propositional expert system. Let furthermore, for some c-atom $A, \Gamma_{1}$ be obtained from $\Gamma$ by omitting (an arbitrary number of) explicit axioms which are in clausal form (not necessarily Horn format) and contain $A$ as positive literal, and $\Gamma_{2}$ by deleting $A$ from (an arbitrary number of) such axioms. Then we have: $\Gamma$ is consistent if and only if $\{A\} \cup \Gamma_{1}$ is consistent or $\Gamma_{2}$ is consistent.

Proof. As to the if-part we remark that $\{A\} \cup \Gamma_{1} \vDash \Gamma$ and $\Gamma_{2} \vDash \Gamma$. As to the only-if-part, note that for every many sorted structure $\Re$ either $k_{\Re} A$ or $k_{\Re} \neg A$. So if $\Re$ is a model of $\Gamma$ then $\pi$ is either a model of $\{A\} \cup \Gamma_{1}$ or of $\Gamma_{2}$, by the definition of $\Gamma_{1}, \Gamma_{2}$.

4.3.4. In our opinion the domain closure axiom is realistic in many applications (apart from the fact that every computer is a finite automaton). The lemmas 3.4-3.8 essentially depend on it. However, Theorem 4.3 remains valid when the domain closure axioms are removed from $E Q$. For, detected contradictions do not depend on the domain closure axioms and, conversely, consistency with the domain closure axiom trivially implies consistency without it.

4.4. Let us briefly discuss the semantical consequences of the conditions (2) and (3) from the previous theorem, since they may slightly deviate from the intended meaning of the knowledge base. It is possible that the consistency of $\Gamma$ essentially depends on the valuation $f\left(c, \ldots, c^{\prime}\right)=$ undefined, i.e. that any valuation $f\left(c, \ldots, c^{\prime}\right)=c_{i}(1 \leqslant i \leqslant n)$ would not yield a model for $\Gamma$. One could say that $f\left(c, \ldots, c^{\prime}\right)=$ undefined possibly saves the expert system from inconsistencies by preventing production rules with occurrences of $f\left(c, \ldots, c^{\prime}\right)$ in the antecedent from firing. Since such rules have obviously not been used in the inference, this may be considered an advantage. On the other hand, this may be considered a disadvantage in cases where $f\left(c, \ldots, c^{\prime}\right)=$ undefined is not realistic (e.g. temperature (patient) $=$ undefined $)$. In these cases we suggest to add the appropriate unit clause $f\left(c, \ldots, c^{\prime}\right)=c_{i}$ and to test consistency again.

4.5. Conceptually speaking it is not difficult to generalize the algorithm from 4.3 to universally quantified expert systems, although some care has to be taken in quantifying $x$ in clauses containing literals of the form $\neg f\left(c, \ldots, c^{\prime}\right)=x$. In these cases only restricted quantification of the form $\forall x \neq$ undefined is allowed. Of course, the algorithm from 4.3 can become very inefficient (from $P\left(c_{0}\right), P\left(c_{1}\right), \neg P\left(x_{i}\right) \vee \cdots \vee \neg P\left(x_{n}\right) \vee Q\left(x_{1}, \ldots, x_{n}\right)$, for instance, $2^{n}$ instances of $Q$ are generated), so we suggest limited use of variables (or, preferably, the use of a more efficient algorithm).

4.6. EXAMPLE. We demonstrate the consistency test in action on the case of 1.2. Taking into account the object tree (sub-objects, single/multivalued attributes) the three rules yield the following clauses in shorthand:

$$
\begin{aligned}
& \neg C(p, c p) \vee P(p, c), \\
& \neg f_{a b d p}(p)=y e s \vee \neg f_{c h a r}(p, p a)=c o \vee \neg P(p, c), \\
& \neg C(p, a p) \vee f_{a b d p}(p)=y e s, \neg P(p, c) \vee f_{a b d p}(p)=\text { yes. }
\end{aligned}
$$

When we add the unit clauses $C(p, c p)$ and $f_{\text {char }}(p, p a)=c o$, the empty clause is easily derived and the algorithm decides to inconsistency. If we only add $C(p, c p)$, then the algorithm decides to consistency on the basis of the following (truth-)valuation:

$$
\begin{aligned}
& C(p, c p)=P(p, c)=T R U E, C(p, a p)=F A L S E, \\
& f_{a b d p}(p)=y e s, f_{\text {char }}(p, p a)=\text { undefined. }
\end{aligned}
$$




\section{EXPRESSIONS}

5.1. The extension of the notion of indexed propositional expert system we discuss in this section aims at supporting arithmetic. We start with a real-life example, again taken from HEPAR. A similar example can be found in [BS], pg. 297.

$$
\text { greater_than 〈biochemistry, }((\text { total_bili-direct_bili)/total_bili) }) \star 100,60\rangle
$$

This example shows an extension of the object-attribute-value representation by allowing attribute expressions instead of only attributes. A straightforward extension of the interpretation of $\langle o, a, v\rangle$ triples into many-sorted predicate logic yields the following atom in shorthand:

$$
((t b(b)-d b(b))) / t b(b)) \star 100>60
$$

In order to capture attribute expressions, the notion of indexed propositional expert system has to be extended. To this end we add new function symbols, called operators and denoted by $o p,+, \ldots$, to our language. Moreover the implicit axioms are extended with the positive diagram of each operator $o p$, i.e. with all defining equations

$$
o p\left(c_{i}, \ldots, c_{j}\right)=c_{f(i, \ldots, j)},
$$

where the constants are assumed to be of the sort that is required by the type of op. Here $f$ does not belong to the language but denotes a function of indices describing the definition of $o p$. Like $=$ and $<$, operators are provided by the system. Let $O P$ denote the set of axioms for operators. A typical example of an operator is addition of integers, denoted by + and written as an infix operator. From now on $I A$ will denote $E Q \cup O \cup O P$.

5.2. We now extend the notion of $c$-atom as defined in Section 3 in the following way: a $c$-atom is a closed atom of the form $P\left(c, \ldots, c^{\prime}\right)$ or of the form:

$$
\text { expression relation symbol expression }
$$

Here a relation symbol is one of the symbols $<,=,>$ and an expression is a term built up from constants and terms of the form $f\left(c, \ldots, c^{\prime}\right)$ by applying the operators (respecting types and sorts).

5.3. From now on indexed propositional expert systems as defined in 3.1 are understood to be extended in the sense of 5.1 and 5.2 above. In order to be able to carry over Theorem 4.3 we introduce the following definition.

Definition. Assume every sort has a constant undefined as stated above in the conditions (2) and (3) of Theorem 4.3. An operator op is called strict if the implicit axioms for op satisfy the following condition:

$$
o p\left(c, \ldots, c^{\prime}\right)=\text { undefined if one or more of the arguments } c, \ldots, c^{\prime} \text { equals undefined. }
$$

Note that for a strict multiplication * of integers we have 0 * undefined $=$ undefined and not $0 *$ undefined $=0$.

5.4. THEOREM. Let $\Gamma$ be an axiomatization of an indexed propositional expert system satisfying the conditions (1)-(4) of Theorem 4.3 above. Assume moreover that the following conditions hold:

(5) Every operator is strict and takes polynomial time.

(6) No operators occur in the positive literals occurring in the explicit axioms of $\Gamma$.

(7) All equations occurring in negative literals in the explicit axioms of $\Gamma$ are of the form expression $=c$.

Then the consistency of $\Gamma$ can be tested in polynomial time. 
Proof. Due to its general formulation, the algorithm from 4.3 carries immediately over to the present case. However, the argument demonstrating the correctness of this algorithm is slightly more complicated, and needs to be restated in some detail. We first note that the evaluation of expressions (such as the example in 5.1) may require more than one unit clause (both the values of $t b(b)$ and $d b(b)$ are necessary). Furthermore, under the conditions (4) and (6) of the theorem, positive literals are of the form $P\left(c, \ldots, c^{\prime}\right), f\left(c, \ldots, c^{\prime}\right)=c^{\prime \prime}, f\left(c, \ldots, c^{\prime}\right)=g\left(d, \ldots, d^{\prime}\right)$, or $c=c^{\prime}$. The latter kind of literals evaluate immediately to TRUE or $F A L S E$ (by using $E Q$ ), so we may assume without loss of generality that they do not occur in the explicit axioms of $\Gamma$. Moreover, under condition (7) of the theorem, negative literals are essentially of the form $\neg P\left(c, \ldots, c^{\prime}\right), \neg$ expression $1<$ expression 2 or $\neg$ expression $=c$. By this more general form of literals involved, as well as the fact that $I A=E Q \cup O \cup O P$ instead of

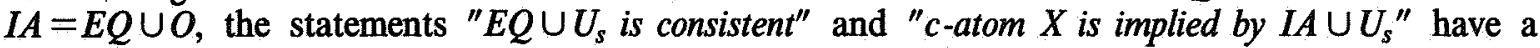
more general meaning than in 4.3. For example, the former statement now also excludes $f(c)=1, f(c)=g(d), g(d)=2 \in U_{s}$, whereas the latter holds for $X$ the $c$-atom from 5.1, with,$- /$, * and $>$ having their usual meaning axiomatized in $I A$, and $t b(b)=100, d b(b)=39 \in U_{s}$. The consistency in the THEN-part follows from the following (truth-) valuation:

$$
\begin{aligned}
P_{i} & = \begin{cases}T R U E & \text { if } P_{i} \in U_{s} \\
F A L S E & \text { otherwise }\end{cases} \\
t_{j} & =\left\{\begin{array}{l}
c \text { if } E Q \cup U_{s} \vDash t_{j}=c \\
\text { undefined otherwise }
\end{array}\right.
\end{aligned}
$$

Again we have that $E Q \cup U_{s}$ is consistent and that every clause not in $U_{s}$ contains a negative literal $L^{-}$whose complement is not implied by $I A \cup U_{s}$. It can happen that $I A \cup U_{s}$ implies $L^{-}$(for example $L^{-}=\neg f(c)+f\left(c^{\prime}\right)<0$, and $f(c)=f\left(c^{\prime}\right), f\left(c^{\prime}\right)=1 \in U_{s}$, with + and $<$ their usual meaning). Then every clause containing $L^{-}$holds in any model of $I A \cup U_{s}$. If neither $L^{-}$nor $L^{+}$is implied by $I A \cup U_{s}$, then we have either $L^{+}=P\left(c, \ldots, c^{\prime}\right) \notin U_{s}$, or $L^{+}$contains a function term $f\left(c, \ldots, c^{\prime}\right)$ such that $E Q \cup U_{s}$ does not imply $f\left(c, \ldots, c^{\prime}\right)=c^{\prime \prime}$ for any constant $c^{\prime \prime}$. By the properties of the constants undefined (conditions (2) and (3) of the theorem), the fact that every operator is strict (condition (5) of the theorem), as well as the syntactic restriction on negative literals (condition (7) of the theorem), the literals $L^{-}$mentioned above are TRUE in the (truth-) valuation. Hence every clause containing $L^{-}$is $T R U E$ in the above (truth-) valuation, which constitutes (in the sense of 4.1) a model of the entire set of clauses.

\section{REMARKS.}

5.4.1. The intuitive idea behind the conditions (1), (4) and (6) is that the positive information, which can be seen as the driving force of the algorithm, should be definite. Omission of (1), (4) or (6) would allow the introduction of incomplete positive information of the form, respectively, $A^{+} \vee B^{+}, f(c)<2$, or $f(c)+f\left(c^{\prime}\right)=3$. The other conditions (2), (3), (5) and (7) concern the simulation of partiality by means of the constants undefined, used for the circumvention of combinatorial explosions that would be caused by the search for consistent function values in a (possibly gigantic) product space of sorts. Thus all conditions are devoted to avoiding combinatorial explosions which would occur in the presence, implicit or explicit, of disjunctions of positive literals.

5.4.2. In Section 4 it is shown that the conditions (1)-(4) cannot be missed. It will be clear that condition (5) of Theorem 2.5, being quite natural, can also not be missed. We shall demonstrate furthermore that neither (6) nor (7) can be missed, by showing that the consistency test becomes NP-hard (see [GJ]) in cases in which (6), respectively (7), do not hold. This will be done in 5.4.3, respectively 5.4.4, by showing that certain disjunctions of positive literals are semantical consequences of indexed propositional expert systems with explicit axioms in Horn format. Then a similar argument as developed in 4.2 , immediately yields the desired result. 
5.4.3. As to (6), let $\beta$ be a sort with exactly two constants, TRUE and FALSE, different from undefined ${ }^{\beta}$. Let eq be a strict equality operator of type $\beta \times \beta \rightarrow \beta$, i.e. eq yields undefined if one or both of its arguments equals undefined and otherwise eq yields TRUE if its arguments are equal, and $F A L S E$ else. It is easily seen that (with $f$ of type $\ldots \rightarrow \beta$ )

$$
E Q \cup O P \cup\{e q(f(c), f(c))=T R U E\} \vDash f(c)=T R U E \vee f(c)=F A L S E .
$$

5.4.4. As to (7), let $\sigma$ be a sort with exactly one constant 0 different from undefined ${ }^{\sigma}$. We trivially have (with $f$ and $f^{\prime}$ of type $\ldots \rightarrow \sigma$ )

$$
E Q \cup\left\{\neg f(c)=f^{\prime}\left(c^{\prime}\right)\right\} \vDash f(c)=0 \vee f^{\prime}\left(c^{\prime}\right)=0 .
$$

At first sight condition (7) seems to be a nasty restriction, since it excludes conditions of the form $f(c)=f^{\prime}\left(c^{\prime}\right)$ in the rules. The reason is that $=$, being reflexive, does not propagate partiality properly (undefined = undefined evaluates to TRUE). There is, however, nothing against using strict equality operators in conditions, i.e. $e q^{\sigma \times a \rightarrow \beta}\left(f(c), f^{\prime}\left(c^{\prime}\right)\right)={ }_{\beta}$ TRUE instead of $f(c)={ }_{\sigma} f^{\prime}\left(c^{\prime}\right)$.

\section{ConcLusion}

We argued how to interpret a rule-based expert system as a many-sorted theory. Then the Tarski semantics yields a well-defined notion of consistency, and theorem proving techniques such as resolution can be used for testing consistency. The relevance for expert systems lies in the fact that inconsistencies make the conclusions of a knowledge-based system highly unreliable. For, what reason do we have to believe a conclusion if its negation could also be derived? We provided means for detecting inconsistencies, but did not discuss how to deal with them. Note that we considered an expert system as a fixed theory, whereas in practice the theory grows during the interaction with a user answering questions posed by the system (in this way the inconsistency of 1.2 was obtained). In our opinion only relatively consistent answers are to be accepted by the system. Starting with a consistent knowledge base we thus maintain consistency as an invariant of the interaction. Recent experiments with a PROLOG implementation of the consistency test and a medical knowledge base show that the models, produced by the consistency test at several moments during the interaction between the user and the system, are very informative.

\section{REFERENCES}

[B] M. BezeM, Consistency of rule-based expert systems. Proceedings of the 9-th Conference on Automated Deduction, Springer Lecture Notes in Computer Science 310, pp. 151-161, SpringerVerlag, Berlin (1987).

[BS] B.G. BUChANAN, E.H. ShORTLIFFe, Rule-based expert systems: the Mycin experiments of the Stanford Heuristic Programming Project. Addison-Wesley, Reading, Massachusetts (1984).

[GJ]M.R. GAREY, D.S. Johnson, Computers and intractability: a guide to the theory of NPcompleteness. Freeman, San Francisco, California (1979).

[IL] T. IMIELINSKI, W. LIPSKI JR., Incomplete information in relational databases. Journal of the ACM 31, 4, pp. $761-791$ (1984).

[L] P.J.F. LuCAS, Knowledge representation and inference in rule-based expert systems. Report CSR8613, Centre for Mathematics and Computer Science, Amsterdam (1986).

[M] J.D. MonK, Mathematical Logic, Springer-Verlag, Berlin (1976).

[R] J.A. RoBINson, Automatic deduction with hyper-resolution. International Journal of Computer Mathematics 1, pp. 227-234 (1965).

[Re] R. REITER, Equality and domain closure in first order databases. Journal of the ACM 27, 2, pp. 235-249 (1980).

[W] C. WALTHER, A many-sorted calculus based on resolution and paramodulation. Pitman, London (1987). 